\title{
SWITCHING POWER SUPPLY FOR INDUCTION ACCELERATORS *
}

\author{
M. Wake ${ }^{\#}$, Y.Arakida, K.Koseki, Y.Shimosaki, K. Takayama, K. Torikai, KEK, Tsukuba, Japan, \\ W. Jiang, K. Nakahiro, Nagaoka University of Technology, Nagaoka, Japan, \\ A.Tokuchi, Nichikon Co.Ltd, Kusatsu, Japan, A. Sugiyama, Shindengen Co.Ltd. Tokyo, Japan.
}

\section{Abstract}

A new particle acceleration method using pulsed induction cells was introduced in the super-bunch project at KEK. Unlike conventional RF acceleration, this acceleration method separates functions of acceleration and confinement. As a result, this acceleration method is capable of accelerating a very long bunch of beam or a wide mass range of particles.

However, it is necessary to give a very fast pulsedexcitation to the magnetic material to induce an electric field to accelerate particles. Switching power supplies of high voltage output with very fast pulse-operation is one of the most important key technologies for this new acceleration method. Features of switching power supply developed for induction synchrotron is reported. The $31 \mathrm{~kW}$ MOS-FET switch performed $1 \mathrm{MHz}$ continuous operation with $15 \mathrm{nsec}$ rise time.

\section{INTRODUCTION}

Synchrotrons traditionally use RF cavities for acceleration device, in which acceleration and confinement of particles are performed at the same time. A new method of acceleration using pulse operated induction cells like induction linear accelerator was first proposed in 2000[1]. The proof of principle (POP) experiment [2] of an induction synchrotron (SI) was performed at KEK in 2006 using 10 induction cells. Since pulse operation of induction cells can separate functions of confinement and acceleration, it gives us much more freedom in beam handling. Any kinds of bucket operations are possible. A particle beam of very long bunch length, "super-bunch"[3] can be accelerated. The velocity range of accelerated beams is very wide thus an all ion accelerator (AIA[4]) can be constructed using SI technology [5],[6].

However, it is necessary to give a very fast pulsedexcitation to the induction cell to perform the acceleration. Switching power supplies of high voltage output with very fast pulse-operation is one of the most important key technologies for this new acceleration method. We have developed a system of $15 \mathrm{~ns}$ rise time $2 \mathrm{kV}$ pulse at continuous repetition rate of $1 \mathrm{MHz}$ using MOS-FET. Induction cells were magnetically modulated for the POP experiment of super-bunch acceleration.

\section{MODULATOR REQUIREMENT}

The acceleration of particle beams has to be repeated for every circulation. Since the KEK-PS has $339 \mathrm{~m}$

\footnotetext{
*Work supported by Grant-In-Aid for Creative Scientific Research (KAKENHI 15GS0217)

\#wake@post.kek.jp
}

circumference, the pulse operation has to be repeated at the rate of $1 \mathrm{MHz}$ continuously. This can be slower in a larger accelerator ring but even faster in a smaller synchrotron. The increase of beam energy per turn has to catch up the ramp rate of the magnets. The excitation voltage of $2 \mathrm{kV}$ for each cell is therefore necessary for reasonable machine operation, if 10 cells are used for acceleration and confinement. Pulse width is typically $400 \mathrm{~ns}$ depending on bunch length, the rise and fall time of the pulse is $15 \mathrm{~ns}$ to achieve beam-handling capabilities. Excitation of the cell has to be bipolar to reset the magnetization of the core. The core material has to have high permeability at high frequency. We used nanocrystalline iron, "finemet", for this purpose [7]. A current of $25 \mathrm{~A}$ is required to excite the magnetic core. As a result, the operation power of the modulator is $31 \mathrm{~kW}$ in average. Such high frequency and high power of switches in continuous operation has never been experienced in other applications.

\section{MODULATOR SYSTEM}

Since the acceleration cells are located at high radiation area, excitation power has to be sent through a transmission line. We used shielded twist pair cable with impedance of $130 \Omega$ as the transmission line. We divided the modulator into FET switch and high voltage DC power supply. DC power supply is placed in the nonradiation area. FET switches are placed in the synchrotron tunnel but hided from high radiation.

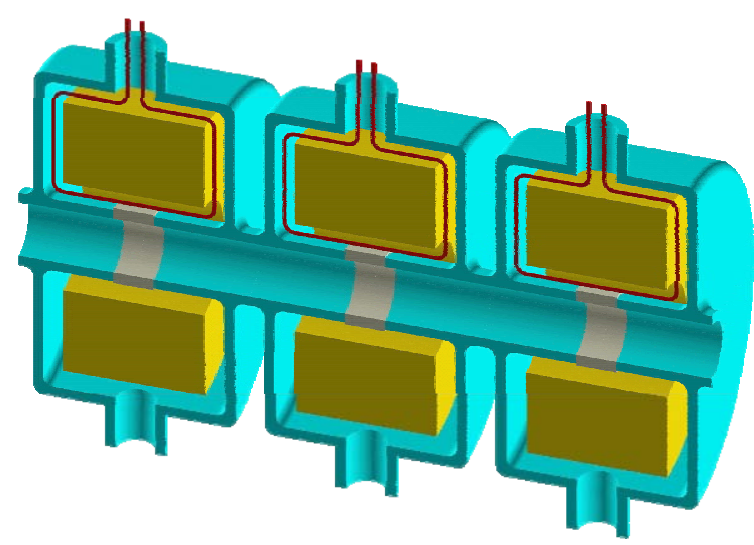

Figure 1: Induction Cell. A toroidal core is excited by a single turn coil to induce electric field in the beam tube. Beam tube has a ceramic gap to prevent eddy current. 
Table 1: Modulator Parameters

\begin{tabular}{l|l}
\hline \hline Output Voltage & $\pm 2500 \mathrm{~V}$ \\
Output Current & $25 \mathrm{~A}$ \\
Pulse Width & $50 \mathrm{~ns}$ to 500ns \\
Repetition & $1 \mathrm{MHz}$ \\
Average Output Power & $31 \mathrm{~kW}$ \\
Rise Time & $15 \mathrm{~ns}$ \\
Time Jitter & $5 \mathrm{~ns}$ \\
Stability & $0.1 \%$ \\
Voltage Ripple & $1 \%$ \\
Operation mode & continuous \\
\hline
\end{tabular}

\section{Power Supply}

The $50 \mathrm{kWDC}$ power supplies are capable of producing $2.5 \mathrm{kV}$ and $20 \mathrm{~A}$. IGBT bridge circuits chop the power input from $3 \phi-400 \mathrm{~V}$ line to produce $20 \mathrm{kHz}$ power. The voltage is stepped up by insulation transformers and rectified into 6 insulated DC voltages. These voltages are used to charge six $0.033 \mu \mathrm{F}$ capacitances connected in series to have 6 times increase of the voltage. The voltage is sent to the accelerator tunnel and the actual power of the system is buffered by a $0.46 \mu \mathrm{F}$ capacitance at the side of the FET switches.

\section{Transmission Line}

The transmission line between FET switch and acceleration cell has impedance $130 \Omega$ and length $60 \mathrm{~m}$. Balanced 2 wires are placed in a shielding braid. This impedance makes the droop of the acceleration voltage inevitable but the droop was in the acceptable range.

\section{Matching Resistance}

A $210 \Omega$ matching resistance was used to adjust the impedance of induction cell to the transmission line impedance. Since, the reflected power is mostly absorbed by the matching resistance, water cooling of the matching resistance is necessary.

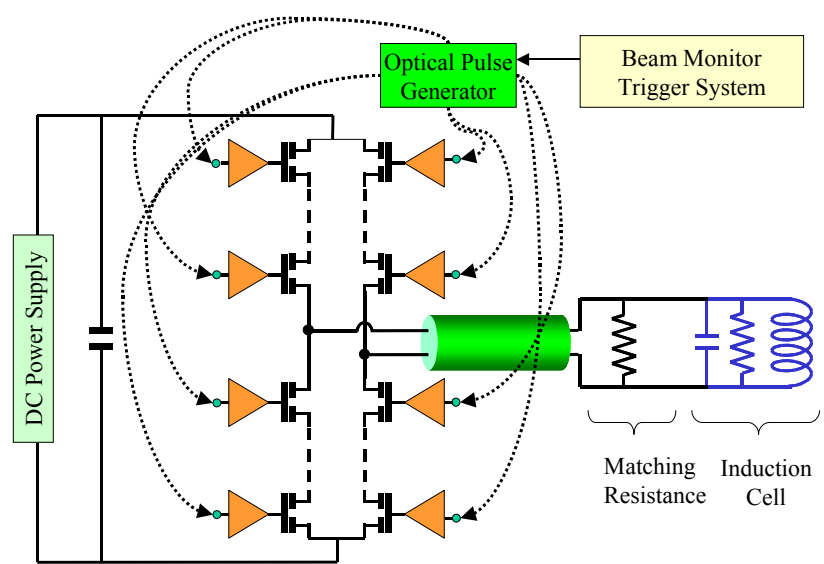

Figure 2: Modulator System. 7 FET's are in series. FET drivers are operated through optical fibre cables. Excitation pulses are sent to the induction cell through a $120 \Omega$ transmission line.

\section{Optical Pulse Generator}

A DSP circuit calculates triggering timing by the beam monitor signal and triggers the optical pulse generator to send gate signals to the FET drivers through optical fibre with keeping electrical isolation to the high voltage circuit.

\section{FET SWITCH}

To achieve bipolar pulse operation, a full bridge circuit of FET switch was designed. Ideally, reset pulse of the acceleration cell can be shorter if the voltage is higher. Using two unipolar switches would give more freedom of operation but the voltage to the FET would be doubled. Full bridge circuit has a limitation of symmetric operation but the number of FET's can be less. Each arms of the full bridge circuit consist of 7 FET's in series to stand for the high voltage of $2 \mathrm{kV}$. IXYS, DE475-102N20A was chosen as the switching FET. Since the switch is operated at very high power and high frequency, the switching loss easily heats up the FET chip. A water-cooled heat sink of copper block was attached to each FET and the driver IC's. The temperature rise of FET was within $40 \mathrm{C}$ in the continuous operation as a result. The inductance of the series circuit was an obstacle for fast switching and caused ringing problems [8]. The use of bus bar connection with inductance canceling strap suppressed the problem. Since the electric potential of each FET in the series are very different, the driving gate signal had to be sent optically through fiber links. Toshiba TODX283 optical transceiver was used for this purpose. The interlock signal is also sent by this transceiver. The potential difference of each FET also causes a problem in the power supplies for the FET drivers. High voltage isolation in DC-DC converter is only guaranteed at low frequency in many cases. A low isolation capacitance is necessary for the operation at high frequency of $1 \mathrm{MHz}$.

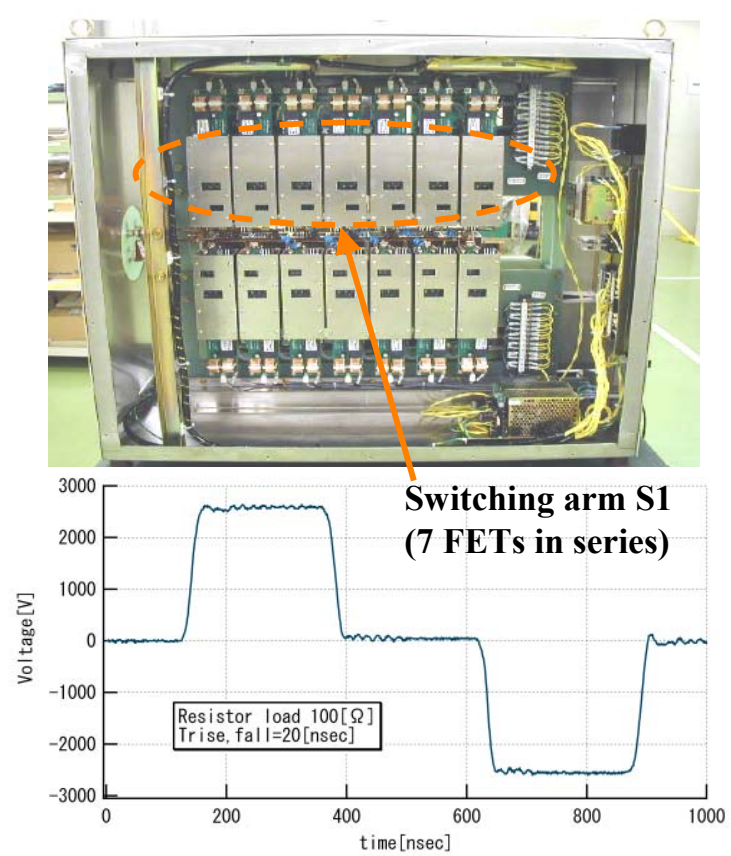

Figure 3: FET Switch and The Switching Wave Form 
We used CD Technology, HB04U with 10pF isolation capacitance. Other cares necessary for the switch is the noise generation. Since a large current is switched at high frequency, switching noises are emitted into the air. Heavy noise emitted from switches would disturb the control of the accelerator system. Each FET module was independently shielded to minimize the noise problems.

\section{FET Voltage Balance}

The isolation capacitance of the driver power supply was found to have a large effect in the voltage sharing among series FET's [9]. The intension of having 7 FET's in series is to divide voltages by factor 7. Because of unequal distribution of voltage, the effectiveness of series connection of the FET's is quite limited in case of large insulation capacitance. FET's are considered as a capacitance, $C_{0}$, in the off state. The isolation capacitance, $C$, effectively connects every stage of series FET to the ground as shown in Fig.4. Then, the voltage across $i$ th FET out of $n$ series is:

$$
V_{i}=\frac{\lambda_{-}^{n-i}-\lambda_{+}^{n-i}}{\lambda_{-}^{n}-\lambda_{+}^{n}} V_{0} .
$$

where,

$$
\lambda_{ \pm}=\frac{C+2 C_{0} \pm \sqrt{C\left(C+4 C_{0}\right)}}{2 C_{0}}
$$

Since FET capacitance is about $200 \mathrm{pF}$, the isolation

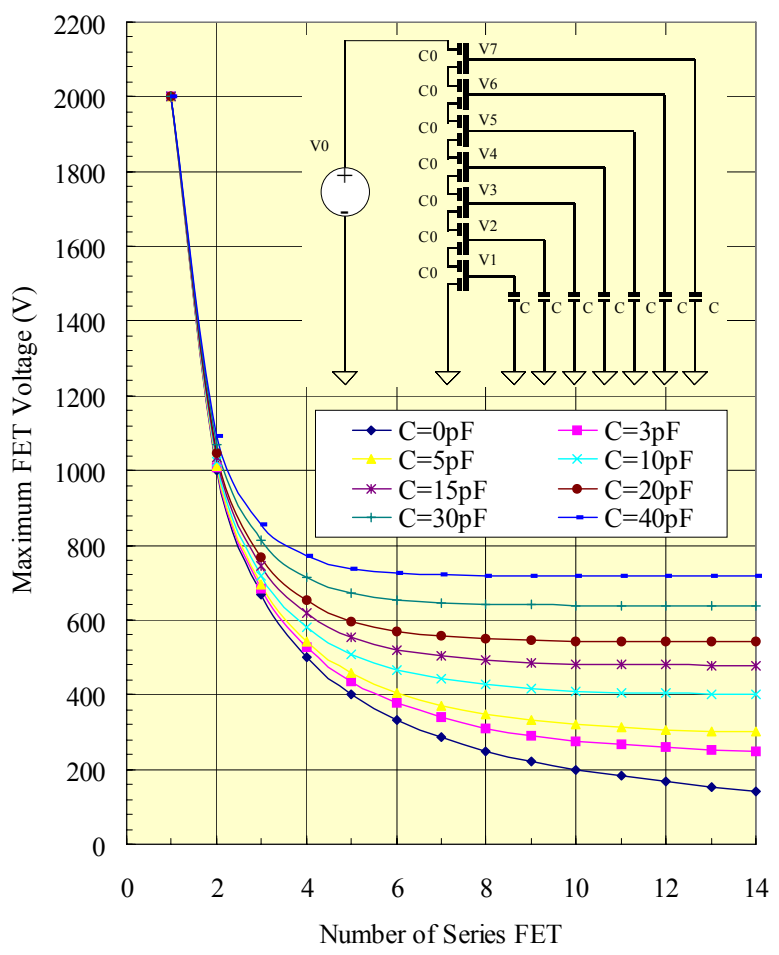

Figure 4: Maximum Drain Source Voltage of the FET. FET's are considered as capacitance $\mathrm{C}_{0}$ in its off state. The potential distribution at each stage can be calculated with isolation capacitance $\mathrm{C}$ of the power supply. Voltage sharing by series FET is only effective when $\mathrm{C}$ is very small. Voltages are calculated for $\mathrm{V}_{0}=2 \mathrm{kV}$ capacitance has to be less than several $\mathrm{pF}$ to make 7 series effective. As an practical solution, parallel capacitances were added to the snubber circuit of each FET to make balance of voltages sacrificing the performance of the switching speed and extra heat generation.

\section{OPERATION RESULTS}

The modulator system of 6 DC power supply and 10 switch units were successfully constructed and operated [10]. Ten induction cells in the KEK-PS were operated as the POP experiment for super-bunch acceleration. The POP experiment showed versatile advantages of induction acceleration and the project is now heading for an all ion accelerator (AIA) with variety of application programs.

\section{FURTHER DEVELOPMENT}

It is desired to have DC-DC converter with low insulation capacitance to use FET's in series to stand for very high voltage. An attempt to realize such power supply had successful result of producing DC-DC converter with $3 \mathrm{pF}$ isolation capacitance using a specially wound transformer with high permeability core. A test operation of FET switch using this DC-DC converter is successfully performed.

A better solution for the switch is not to use series connection but to use a device that can handle very high voltage. SiC-FET is a candidate for that purpose. Such devices are under development but preliminary results using SiCED product achieved $1 \mathrm{kV}$ and $5 \mathrm{~A}$ switching at frequency of $1 \mathrm{MHz}$ though the operation is still in a burst mode [11]. Si-Thiristor can be used with even greater powers. Preliminary results showed easy $2 \mathrm{kV}$ operation but it has 50 ns gate delay and takes 100 ns to turn-off [11].

\section{CONCLUSION}

The modulator system for induction synchrotron was successfully constructed with $2 \mathrm{kV} 25 \mathrm{~A}$ output and continuously operated in the POP experiment at the repetition rate of $1 \mathrm{MHz}$. Further development to achieve AIA operation is now under way [12].

\section{REFERENCES}

[1] K.Takayama and J.Kishiro, N.I.M. A 451, 304(2000).

[2] K.Takayama et al., Phys. Rev. Lett. 94, 144801(2005).

[3] K.Takayama et al.,Phys. Rev. Lett. 88, 144801(2002)

[4] K.Takayama et al., Phys. Rev. Lett. 98, 054801(2007).

[5] T.Iwashita et al., in this proceedings, TUPAN044.

[6] K.Takayama et al., in this proceedings, TUXC02.

[7] K.Torikai et al., KEK Preprint 2005-80, (2005).

[8] K. Koseki et al., IEEJ Trans. FM, 126, 121 (2006).

[9] K. Koseki et al., NIM A554, 64(2005).

[10] Y. Shimosaki et al., in this proceedings, TUPAN050.

[11] W. Jiang et al., Proc. of RPIA2006, 78 (2006).

[12] E. Nakamura et al., in this proceedings, TUPAN046. 